STI rates at that time, it is improbable that its use has continued to affect rates to such an extent. The effects of erectile dysfunction and club drugs, sexual health education, and sexual practises on national STI rates have yet to be confirmed; more research is needed into these potential contributors. National efforts to combat the rising rates of STI include educational and clinical guidelines to prevent and control their spread.

\section{P3.077 SOCIODEMOGRAPHIC AND CLINICAL PROFILE OF WOMEN WITH GENITAL INFECTION BY THE HUMAN PAPILLOMAVIRUS SEEN AT A BRAZILIAN REFERENCE HOSPITAL}

doi:10.1136/sextrans-2013-051184.0537

'J Gaspar, 'E Gir, 'R K Reis, ${ }^{2}$ S M Quintana. 'Nursing School of Ribeirão Preto, University of São Paulo, Ribeirão Preto, Brazil; ${ }^{2}$ Faculty of Medicine of of Ribeirão Preto, University of São Paulo, Ribeirão Preto, Brazil

Introduction Infection by the human papillomavirus (HPV) is rated as a public health issue, and is considered to be the most common sexually transmitted disease (STD). It is estimated that approximately 600 million people in the world carry HPV, and that 75 to $80 \%$ will become infected with HPV some time in their lifetime. Therefore, the objective of this study was to analyse the sociodemographic and clinical aspects of women with genital infections by HPV.

Methodology This descriptive, quantitative study was performed at the Gynecology and Obstetrics Clinic for Infectious Diseases (Serviço de Moléstias Infecciosas em Ginecologia e Obstetrícia SEMIGO) of the University Hospital of the Ribeirão Preto Faculty of Medicine, University of São Paulo (HCFMRP-USP), located in Ribeirão Preto, São Paulo, Brazil. The study included a total of 1027 women diagnosed with genital infection by HPV, which could be present in the form of low-grade squamous intraepithelial lesion (LSIL), high-grade squamous intraepithelial lesion (HSIL) or warts (vulvar, vaginal, cervical and perianal).

Results The predominant age group was between twenty and twenty-nine years of age, 387 (37.7\%); 662 (64.4\%) were of white ethnicity; 597 (58.1\%) were married or lived with a partner; 438 $(42.6 \%)$ had five to eight years of education; and $529(51.5 \%)$ were unemployed. Regarding the type of lesion, 506 (49.3\%) women had warts, $398(38.8 \%)$ LSIL and 390 (38.0\%) HSIL. Most did not consume alcohol (880 or $85.7 \%$ ), tobacco (711 or $69.2 \%$ ) or any other illicit drug (880 or $85.7 \%)$. Of all subjects, $702(68.3 \%)$ tested negative for HIV, 823 (80.1\%) negative for HBsAg, 777 (75.7\%) negative for HCV, and $832(81.0 \%)$ negative for VDRL.

Conclusion Women must be provided with access to high quality preventive tests through efficient mass screening programmes, and it must be ensured they receive clinical follow-up.

\section{P3.078 PREVALENCE OF STI/STDS AMONG WOMEN OF REPRODUCTIVE AGE GROUP IN TRIBAL DISTRICT OF NORTH INDIA}

doi:10.1136/sextrans-2013-051184.0538

GUPTA A. I G Medical College Shimla, Shimla, India

Research question What is the prevalence of RTIs/STDs among women of reproductive age group in a district of Kinnaur Himachal Pradesh Objectives (1) To estimate the prevalence of RTI/STI among women of reproductive age by using the syndromic case definition. (2) To identify the common signs and symptoms ofRTI/ STI among women (3) To find out other socio-demographic variables associated with causation of RTI/STI in women.
Study design Exploratory in nature with application of quantitative and qualitative research methodology.

Setting 60 clusters, comprising of villages in rural area and localities in urban area of district Kinnaur Himachal Pradesh North India.

Participants 600 ever married women in the reproductive age group (i.e. $15-45$ years).

Study period One year (Feb, 2011- Jan, 2012).

Study variables Age, literacy status, number of live children, symptoms (accrding to the syndromic approach).

Statistical analysis Percentages, Chi square test.

Results Prevalence of RTI/STI came out to be $39.2 \%$ with a higher rural (44\%) than urban (32\%). Almost half of the symptom-positive women were of 25-34 years of age. Prevalence of RTI/STI was found to be highest among women with 1 or 2 live children $(26.7 \%$ and $30.00 \%)$ in rural and urban areas respectively. The commonest symptom of RTI/STD was vaginal discharge (91\%) followed by lower abdominal pain $(64 \%)$. Other common associated symptoms were backache (76\%), vulval itching (51\%) and burning during urination (34\%).

Conclusion In view of the higher prevalence of RTI/STD among rural and illiterate women primary health care level needs to be strengthened in respect of reproductive health, and awareness about reproductive health issues should be raised through suitable communication in order to bring about a positive behaviour change.

\section{P3.079 PREVALENCE AND COFACTORS FOR STIS AMONG PREGNANT ADOLESCENTS IN WESTERN KENYA}

doi:10.1136/sextrans-2013-051184.0539

'A L Drake, ${ }^{2} \mathrm{~J}$ Kinuthia, ${ }^{2} \mathrm{D}$ Matemo, 'R S McClelland, 'J Unger, 'G John-Stewart. ${ }^{1}$ University of Washington, Seattle, WA, United States; ${ }^{2}$ University of Nairobi, Nairobi, Kenya

Background STIs among pregnant women contribute maternal and infant morbidity. There are limited data on STIs among pregnant adolescents. We determined and compared prevalence and correlates of STIs among pregnant adolescent and adult women in Western Kenya.

Methods HIV-1 negative women were enrolled during pregnancy. Demographic and clinical characteristics and blood and genital samples were collected. Chlamydia trachomatis (CT) and Neisseria gonorrhoea (GC) were detected using endocervical nucleic acid amplification, syphilis by rapid plasma reagin tests, and trichomonas vaginalis (TV) by wet preparations. Analyses were restricted to adolescents (age 14-21) and adults (age > 21) with STI data.

Results Among 1156 HIV-1 negative pregnant women, median age was 22 years and 537 (46\%) were adolescents. Adolescents were less likely to be married (64\% vs. $92 \%$; p < 0.001$)$ and more likely to have traded sex (13\% vs. $3 \%$; $p=0.05)$; adolescents and adults had similar prevalence of prior STIs (7\%), vaginal drying (18\%) and unprotected sex during past month (56\%). The prevalence of STIs was: CT, $5 \%$; GC, $2 \%$; TV, $6 \%$; and syphilis $1 \%$. Compared to adults, risk of CT (odds ratio $(\mathrm{OR})=3.23$; 95\% Confidence interval $(\mathrm{CI})=1.83-7.73)$, GC $(\mathrm{OR}=1.87,95 \% \mathrm{CI}=0.84-4.16)$, and $\mathrm{TV}$ $(\mathrm{OR}=1.61,95 \% \mathrm{CI}=1.00-2.60)$ were higher among adolescents, but syphilis risk was lower $(\mathrm{OR}=0.25,95 \% \mathrm{CI}=0.05-1.17)$. Among adolescents, CT risk factors included ever trading sex $(\mathrm{aOR}=2.71$, $95 \% \mathrm{CI}=1.06-13.33)$. Among adults, TV risk factors included vaginal drying and lifetime number of sex partners; CT risk factors included vaginal drying and younger age. History of STI was a risk factor for syphilis among both adolescents and adults. No risk factors were identified for gonorrhoea.

Conclusion Prevalences of CT, GC, and TV were significantly higher among adolescents than adults in this pregnant cohort. Trading sex was more prevalent in adolescents and was associated with a 2.7-fold increased risk for CT among adolescents. Tailoring pregnancy STI screening approaches for adolescents may be useful. 